\title{
BMJ Open Quality of life, loneliness and health- related characteristics among older people in Liaoning province, China: a cross-sectional study
}

Yaxin Zhu, ${ }^{1}$ Jie Liu, ${ }^{2}$ Bo $Q u,{ }^{2}$ Zhe Yi $^{3}$

To cite: Zhu Y, Liu J, Qu B, et al. Quality of life, loneliness and health-related characteristics among older people in Liaoning province, China: a crosssectional study. BMJ Open 2018:8:e021822. doi:10.1136/ bmjopen-2018-021822

- Prepublication history for this paper is available online. To view these files, please visit the journal online (http://dx.doi org/10.1136/bmjopen-2018021822).

$\mathrm{BQ}$ and $\mathrm{ZY}$ contributed equally.

Received 19 January 2018 Revised 9 August 2018 Accepted 2 October 2018

D) Check for updates

(C) Author(s) (or their employer(s)) 2018. Re-use permitted under CC BY-NC. No commercial re-use. See rights and permissions. Published by BMJ.

${ }^{1}$ Department of Social Medicine, School of Public Health, China Medical University, Shenyang, People's Republic of China ${ }^{2}$ Department of Health Statistics, School of Public Health, China Medical University, Shenyang, People's Republic of China

${ }^{3}$ Department of Prothodontics, School of Stomatology, China Medical University, Shenyang, People's Republic of China

Correspondence to

Dr Zhe Yi;

zheyi_2000@sina.com

\section{ABSTRACT}

Objectives The aim of the study was to examine the relations among quality of life (QOL), loneliness and healthrelated characteristics in a sample of Chinese older people. Design Cross-sectional study.

Setting Communities in Dandong city, Liaoning province, China.

Participants Sample of 732 older people aged 60 and older who were living in Dandong, Liaoning province, China.

Methods A questionnaire was administered to the participants face-to-face. The questionnaire contained four sections: demographic characteristics, health-related characteristics, the EQ-5D Scale and the UCLA Loneliness Scale. The t-test, F-test and multivariable linear regression analyses were performed to individually test associations between the demographic data, health-related characteristics, Ioneliness and QOL.

Results Chronic diseases, loneliness, age and smoking status were negatively associated with QOL $(p<0.05)$. Satisfaction with health services, income and physical activity were positively associated with Q0L $(p<0.05)$. Conclusions Loneliness, chronic diseases and health service satisfaction were important factors related to low QOL among older people in China. The findings indicate that reducing loneliness, managing chronic diseases and improving the health service may help to improve the $\mathrm{QOL}$ for older people.

\section{INTRODUCTION}

The global population is ageing rapidly. In 1950 , only $8 \%$ of the global population was $\geq 60$ years of age. ${ }^{2}$ The HelpAge Global Network 2015 review reported that $12 \%$ of the global population was $\geq 60$ years of age ${ }^{3}$ and that by 2050 , this percentage would double. $^{13}$ The People's Republic of China has the largest population $\geq 60$ years old (hereafter designated older people) in the world and in 2014 accounted for $14.4 \%$ of the total Chinese population. ${ }^{4}$ The percentage of older people in China is expected to reach $33.9 \%$ by $2050 .{ }^{3}$ In addition, the Chinese older population currently constitutes one-fifth of the total worldwide older people

\section{Strengths and limitations of this study}

- Hierarchical linear regression analysis was performed to provide a conceptual and statistical mechanism for investigating and drawing conclusions regarding the different levels of associated factors (Ioneliness, heath-related characteristics and demographic characteristics) of quality of life (QOL) among older people.

- A stratified random sampling design was used in this study to improve the sample's representativeness.

- Standardised instruments (EQ-5D and UCLA) assessing the QOL and loneliness were used to make the results more reliable.

- This study was an observational investigation employing a cross-sectional design to explore the relation between QOL and loneliness, which may limit the ability to confirm a causal relation.

population. ${ }^{2}$ Notably, older people are more vulnerable to chronic diseases, ${ }^{5}$ and given the rapid growth of the ageing population, the prevalence of chronic diseases is expected to increase among older people. ${ }^{5}$ Hence, the ageing population should have a profound impact on healthcare systems and economic growth in China and worldwide. ${ }^{12}$

Maintaining older people's good health positively impacts both them and society in general, as the availability of human and social resources for the aged strongly depends on their health status. ${ }^{1}$ However, healthy ageing involves more than the absence of disease. ${ }^{1}$ A report by the WHO has suggested that improving quality of life (QOL) for older people should be the outcome of realising the policy framework of 'active ageing. ${ }^{6}$ Adding 'quality' to older people's life to improve their health, social function, independence and activity has become the goal of a prolonged life. ${ }^{7}$ QOL is an assessment of health status based on a medical model that reflects an individual's physical, psychological and sociological health. ${ }^{8}$ In addition, it has 
been recommended that disease-centred curative health systems embrace integrated care focusing on the needs of older people. ${ }^{1}$ Thus, assessment of QOL is seen as an essential element in the care of older people,${ }^{9-11}$ and improving their QOL has become a prioritised element in their medical care. ${ }^{6}$ Identifying the factors associated with QOL is needed if new interventions that will lead to improved QOL among older people are to be developed. Some health-related characteristics, such as chronic diseases and physical activity, associated with QOL among older people have been well documented. ${ }^{12} 13$

Loneliness among older people is a common ${ }^{14}$ and serious problem. ${ }^{15}$ The relation between loneliness and health has been recognised among older people within the past decade, with loneliness being related to adverse physical and mental health outcomes. ${ }^{141617}$ Due to recent social and economic changes in China, younger people have migrated or emigrated to relatively economically developed areas to seek employment and a better life. ${ }^{18}$ Also, China is a country that values the culture of collectivism and filial piety. Older people are still expected to be provided with care by their children. Therefore, the perception of the gap between expected and actual amounts of support that older people received from their families would be stronger for older people. ${ }^{15}$ This perceived gap would be the reason why Chinese older people had serious problems of loneliness.

Although QOL, as a good measure of overall health, has also been reported to be influenced by loneliness among older people in certain countries, such as Sweden ${ }^{19}$ and the Netherlands, ${ }^{20}$ the relation between QOL and loneliness among older people in China has not been fully assessed to date. ${ }^{15}$ It is also suggested that interventions on lonely older people may improve well-being and lengthen life. ${ }^{21}$ Hence, the relation between QOL and loneliness should be fully investigated to provide implications for proposing new interventions on improving QOL among Chinese older people. The purpose of the study was to examine the relations among QOL, loneliness and health-related characteristics in a sample of Chinese older people.

\section{MATERIALS AND METHODS}

\section{Study sample and procedures}

Older age is generally defined in relation to retirement from paid employment and receipt of pensions. ${ }^{22}$ The age of 60 is roughly equivalent to retirement ages in China. In this study, 60 years of age was chosen as the cut-off point for defining older people. This study employed a cross-sectional design with a stratified random sampling method on older people of age $\geq 60$ years in Dandong city, Liaoning province. Compared with other cities in China, Dandong has more older people. In 2014, those aged $\geq 60$ years accounted for $21.6 \%$ of the Dandong population, and this percentage ranked third in Liaoning province.

Dandong has six districts. One community in each district was randomly selected to achieve the required sample size, which was determined by the criterion that the sample size should be 20 times the number of variables. In this study, the questionnaire contained 36 variables, and the required sample size was 720 . Inclusion criteria were age $\geq 60$ years, residence in Dandong, having provided the written informed consent, and ability to understand the questionnaire and communicate. Those who refused to participate in the study were excluded. Additionally, older people who were determined by the clinician to have severe physical conditions affecting daily life and unsuitable to take part in the study were also excluded. During this survey, a questionnaire was administered by face-to-face interview by trained investigators with the participants from February 2017 to July 2017. If the participants had any problem with completing the survey, the investigators assisted them.

In total, 784 eligible participants were approached in this survey, and 764 completed the questionnaires (completion rate $97.4 \%$ ). When a participant did not answer $>20 \%$ of the questions, the associated questionnaire was not included in the study. After exclusion of 32 invalid questionnaires, 732 questionnaires were identified as valid (response rate $93.4 \%$ ).

\section{Measurements}

The questionnaire contained four sections: demographic characteristics, heath-related characteristics and the EQ-5D Scale and the UCLA Loneliness Scale. The demographic characteristics were age, gender, marital status, education, monthly income, place of residence (urban or rural) and living arrangement (empty or non-empty nester). Empty nesters were older people who never had children or whose children had left home such that empty nesters were defined as those living alone or with only a spouse. ${ }^{23}$

Heath-related characteristics consisted of smoking status, physical activity status, chronic disease status and satisfaction with health services. To assess the level of physical activity, participants were asked if they exercised at least six times a week. ${ }^{24}$ Respondents were defined as having a chronic disease(s) if they had been diagnosed by a health professional(s). Satisfaction with health services was evaluated using one item from WHOQOL-BREF, ${ }^{25}$ in which respondents rated the scale from 1 (very dissatisfied) to 5 (very satisfied).

The EQ-5D was used to evaluate QOL of the participants, which is a generic tool developed by the EuroQol Group. $^{26}$ The EQ-5D contains five items covering different dimensions (mobility, self-care, usual activities, pain/discomfort and anxiety/depression). Each item has three possible responses (no problem, moderate problem or extreme problem). A single EQ-5D summary index score ranging from -0.149 to 1.0 was calculated using the Chinese time trade-off model. ${ }^{26}$ Higher values implied better QOL. In addition, the EQ-5D also contains the Visual Analogue Scale (VAS). The VAS was used to assess the respondents' own perceptions of their health status on a scale of 0 (worst) to 100 (best). The EQ-5D has 
been shown to be applicable in China for Chinese older people. ${ }^{24}$ 27-29 The reliability of the EQ-5D for our study was acceptable with a Cronbach's alpha of 0.82.

Loneliness in older people was assessed with the use of the UCLA Loneliness Scale, ${ }^{30}$ which has 20 items rated on a 1-point to 4-point, Likert-type Scale. The total UCLA score ranges from 20 to 80 , with greater scores defining greater degrees of loneliness. The scale ranges are the following: 20-34, a low loneliness level; 35-49, a moderate loneliness level; 50-64, a moderately high loneliness level; and $65-80$, a high loneliness level. ${ }^{30}$ We found the scale to be reliable, as the Cronbach's alpha was 0.84 .

\section{Statistical analyses}

The Student's t-test, F-test and hierarchical linear regression analysis were performed to determine the association between QOL and the demographic data, health-related factors and the UCLA Loneliness Scale. In block 1, demographic characteristics (age, gender, marital status, education, monthly income, place of residence and living arrangement) were included in the model. Health-related characteristics (smoking status, physical activity status, chronic disease status and satisfaction with health services) were added in block 2. In block 3, loneliness was added. Variances of QOL explained by different groups of independent variables were examined by $\Delta \mathrm{R}^{2}$ (R squared change). Data were analysed using SPSS V.21 for Windows. A p value $<0.05$ was considered to be statistically significant.

\section{Patient and public involvement}

Neither participating older people were involved in setting the research questions or the outcome measures, nor were they involved in developing plans for the design or implementation of the study. The participants were informed that the results were to be published in an open access journal.

\section{RESULTS}

\section{Participant characteristics}

The demographic characteristics of the participants are described in table 1. A total of 375 women (51.2\%) and 357 men $(48.8 \%)$ participated in the study. Their ages ranged from 60 to 96 years with a mean age of $71.34 \pm 7.73$ years (SD); 241 of the participants $(32.9 \%)$ lived in rural areas. Approximately one-half of the participants $(341,46.6 \%)$ were empty nesters. The F-test or t-test revealed that there were significant differences in the QOL scores for participants of different ages, residences, living arrangements, monthly incomes and education levels $(\mathrm{p}<0.05)$. Participants who were younger, lived in an urban area or were not empty nesters had higher QOL scores (table 1).

Regarding health-related characteristics (table 2), the majority of the participants $(559,76.4 \%)$ suffered from a chronic disease(s), and approximately one-half $(436,59.5 \%)$ were satisfied or very satisfied with the health services that they received. For the heath-related
Table 1 Relationship between demographic characteristics and QOL $(\mathrm{n}=732)$

\begin{tabular}{|c|c|c|c|}
\hline Variable & $\begin{array}{l}\text { Participants, } \\
\text { n (\%) }\end{array}$ & $\begin{array}{l}\text { EQ-5D } \\
\text { index score }\end{array}$ & $\mathbf{F} / \mathbf{t}$ \\
\hline \multicolumn{4}{|l|}{ Age† } \\
\hline $60-69$ & $289(39.5)$ & $0.95 \pm 0.10$ & $8.149^{\star \star}$ \\
\hline $70-79$ & $345(47.1)$ & $0.92 \pm 0.12$ & \\
\hline$\geq 80$ & $98(13.4)$ & $0.90 \pm 0.15$ & \\
\hline \multicolumn{4}{|l|}{ Gender $\neq$} \\
\hline Male & $357(48.8)$ & $0.93 \pm 0.12$ & 0.301 \\
\hline Female & $375(51.2)$ & $0.93 \pm 0.12$ & \\
\hline \multicolumn{4}{|l|}{ Residenceł } \\
\hline Rural & 241 (32.9) & $0.92 \pm 0.12$ & $-2.333^{*}$ \\
\hline Urban & $491(67.1)$ & $0.94 \pm 0.12$ & \\
\hline \multicolumn{4}{|l|}{ Living arrangementł } \\
\hline Empty nester & $341(46.6)$ & $0.92 \pm 0.13$ & $2.221^{*}$ \\
\hline Non-empty nester & $391(53.4)$ & $0.94 \pm 0.11$ & \\
\hline \multicolumn{4}{|l|}{ Marital status $†$} \\
\hline Single & $18(2.4)$ & $0.97 \pm 0.10$ & 2.304 \\
\hline Married & $688(94.0)$ & $0.93 \pm 0.12$ & \\
\hline Divorced/widowed & $26(3.6)$ & $0.89 \pm 0.14$ & \\
\hline \multicolumn{4}{|l|}{ Monthly income† } \\
\hline$<2000$ & $367(50.1)$ & $0.90 \pm 0.14$ & $21.722^{* \star}$ \\
\hline 2000-3000 & $194(26.5)$ & $0.95 \pm 0.08$ & \\
\hline$>3000$ & $171(23.4)$ & $0.97 \pm 0.09$ & \\
\hline \multicolumn{4}{|l|}{ Education† } \\
\hline $\begin{array}{l}\text { Primary school or } \\
\text { below }\end{array}$ & $323(44.1)$ & $0.92 \pm 0.13$ & $3.236^{\star}$ \\
\hline Junior high school & $294(40.2)$ & $0.95 \pm 0.10$ & \\
\hline Senior high school & 89 (12.2) & $0.94 \pm 0.11$ & \\
\hline College and above & $26(3.5)$ & $0.92 \pm 0.12$ & \\
\hline $\begin{array}{l}{ }^{*} \mathrm{p}<0.05 ;{ }^{* *} \mathrm{p}<0.01 . \\
\text { †t-test. } \\
\text { † F-test. } \\
\text { QOL, quality of life. }\end{array}$ & & & \\
\hline
\end{tabular}

characteristics, the F-test or t-test results showed that significant differences existed in the QOL scores as related to chronic disease status, smoking status, physical activity and satisfaction with health services $(\mathrm{p}<0.05)$. The participants who had a chronic disease (s), smoked, were not physically active, or were dissatisfied with their health services had lower QOL scores (table 2).

\section{Quality of life}

The mean EQ-5D index score for the participants was $0.93 \pm 0.12$ (range, $0.22-1.00$ ). The mean VAS score was $81.25 \pm 14.38$ (range, $0-100$ ). The distribution of the EQ-5D Scale scores is presented in table 3. The most prevalent health-related problem reported by the participants was pain/discomfort (196, 26.8\% had moderate or extreme pain/discomfort). 
Table 2 Relationship between health-related characteristics and QOL $(n=732)$

\begin{tabular}{|c|c|c|c|}
\hline Variable & $\begin{array}{l}\text { Participants, } \\
\text { n (\%) }\end{array}$ & $\begin{array}{l}\text { EQ-5D } \\
\text { index score }\end{array}$ & $F / t$ \\
\hline \multicolumn{4}{|l|}{ Chronic disease $†$} \\
\hline Yes & $559(76.4)$ & $0.87 \pm 0.15$ & $-7.158^{\star *}$ \\
\hline No & $173(23.6)$ & $0.95 \pm 0.10$ & \\
\hline \multicolumn{4}{|l|}{ Smoking status $†$} \\
\hline Yes & $220(30.1)$ & $0.92 \pm 0.13$ & $-1.984^{*}$ \\
\hline No & $512(69.9)$ & $0.94 \pm 0.11$ & \\
\hline \multicolumn{4}{|l|}{ Physical activity $\dagger$} \\
\hline No & $633(86.5)$ & $0.93 \pm 0.12$ & $-2.631^{\star *}$ \\
\hline Yes & 99 (13.5) & $0.95 \pm 0.09$ & \\
\hline \multicolumn{4}{|l|}{ Health services $\ddagger$} \\
\hline Very dissatisfied & $20(12.7)$ & $0.83 \pm 0.21$ & $29.230^{\star *}$ \\
\hline Dissatisfied & $53(22.3)$ & $0.86 \pm 0.14$ & \\
\hline $\begin{array}{l}\text { Neither satisfied } \\
\text { nor dissatisfied }\end{array}$ & $223(15.5)$ & $0.89 \pm 0.13$ & \\
\hline Satisfied & 353 (38.2) & $0.96 \pm 0.08$ & \\
\hline Very satisfied & 83 (11.3) & $0.97 \pm 0.09$ & \\
\hline
\end{tabular}

${ }^{*} \mathrm{p}<0.05$.

${ }^{* *} \mathrm{p}<0.01$

†t-test.

$\ddagger$ F-test.

QOL, quality of life.

\section{Loneliness}

The mean UCLA loneliness score was $40.73 \pm 8.73$ (range, 20-64). According to their loneliness scores, 547 participants $(74.7 \%)$ had a moderate or a moderately high loneliness score (table 4). A significant difference was found for the QOL scores for groups with different degrees of loneliness $(p<0.001)$. Participants with moderately high loneliness scores had lower QOL scores $(0.86 \pm 0.17)$ compared with participants in the other two groups. The relationships between their QOL and loneliness scores are listed in table 4.

\section{Relation between the demographic data, health-related} factors, Ioneliness and QOL

Hierarchical linear regression analyses of the factors associated with QOL are presented in table 5. In block 1,

\begin{tabular}{|c|c|c|c|}
\hline Dimension & $\begin{array}{l}\text { No } \\
\text { problem, } \\
\text { n (\%) }\end{array}$ & $\begin{array}{l}\text { Moderate } \\
\text { problem, } \\
\text { n (\%) }\end{array}$ & $\begin{array}{l}\text { Extreme } \\
\text { problem, } \\
\mathrm{n}(\%)\end{array}$ \\
\hline Mobility & 678 (92.6) & $53(7.2)$ & $1(0.1)$ \\
\hline Self-care & 713 (97.4) & $14(1.9)$ & $5(0.7)$ \\
\hline Usual activities & 677 (92.5) & $55(7.5)$ & $0(0)$ \\
\hline Pain/discomfort & 536 (73.2) & $193(26.4)$ & $3(0.4)$ \\
\hline Anxiety/depression & $610(83.3)$ & $121(16.5)$ & $1(0.1)$ \\
\hline
\end{tabular}

Table 4 Relationship between loneliness and QOL $(n=732)$

Participants, EQ-5D index

\begin{tabular}{lrll} 
Loneliness level & $\mathbf{n}(\%)$ & score & F \\
\hline Low & $185(25.3)$ & $0.97 \pm 0.07$ & $33.889^{* * *}$ \\
Moderate & $449(61.3)$ & $0.93 \pm 0.11$ & \\
Moderately high & $98(13.4)$ & $0.86 \pm 0.17$ &
\end{tabular}

${ }^{\star * \star} \mathrm{p}<0.001$.

QOL, quality of life.

age, income, education, living arrangement and marital status were significantly associated with QOL $(\mathrm{p}<0.05)$. When health-related characteristics were added in block 2, age, income, chronic disease(s), health service satisfaction, smoking and physical activity were significantly associated with QOL $(\mathrm{p}<0.05)$. In block 3, among these demographic and health-related characteristics, having a chronic disease(s), age of the participant and smoking were negatively associated with QOL $(p<0.05)$, with chronic disease having standardised coefficients of -0.234 . Satisfaction with health services, higher income, and being physically active were positively associated with the QOL of the participants $(\mathrm{p}<0.05)$. In addition, loneliness was significantly and negatively associated with QOL (level=moderate: $\beta=-0.190, p<0.05$; level=moderately high: $\beta=-0.260, p<0.05$; reference group: level=low). Loneliness explained 5.2\% of the variance of QOL.

\section{DISCUSSION}

This study contributed to our knowledge concerning the relation between QOL, loneliness and health-related characteristics among older people living in Dandong city, Liaoning province, China. Our results indicated that the pain/discomfort facet of QOL was the most prevalent problem among the participants. This finding is in line with previous studies on Chinese ${ }^{2728}$ and Vietnamese older people. ${ }^{31}$ One population-based study revealed that more than one-half of older Americans had been bothered by pain in the month prior to being questioned. ${ }^{32}$ The older people often experience chronic pain, ${ }^{33}$ which is a disability that can cause decreased mobility and depression. ${ }^{32}$ These factors may increase healthcare costs and may decrease productivity. ${ }^{33}$ Bhattarai and Phillips suggested that cost-efficient intervention strategies for management of the pain in the older population should be a priority for healthcare providers. ${ }^{33}$

We found loneliness to be significantly correlated with the QOL of our participants, and those who had a greater degree of loneliness also reported a poorer QOL. Loneliness may impair the immune and cardiovascular systems. ${ }^{14}$ The negative effects of loneliness on the physical and psychological health of the older people have been widely documented. ${ }^{14}{ }^{17}$ Lonely individuals are also more likely to engage in behaviour that results in poor health outcomes. ${ }^{34}$ These factors may all contribute to and impair QOL for older people. ${ }^{9}$ We found that $74.7 \%$ 
Table 5 Hierarchical linear regression analyses of the factors associated with QOL $(n=732)$

\begin{tabular}{|c|c|c|c|}
\hline Factor & $\begin{array}{l}\text { Block } 1 \\
(\beta)\end{array}$ & $\begin{array}{l}\text { Block } 2 \\
(\beta)\end{array}$ & $\begin{array}{l}\text { Block } 3 \\
(\beta)\end{array}$ \\
\hline \multicolumn{4}{|l|}{ Age† } \\
\hline $70-79$ & $-0.081^{*}$ & $-0.074^{*}$ & $-0.079^{*}$ \\
\hline$\geq 80$ & -0.077 & -0.043 & -0.050 \\
\hline \multicolumn{4}{|l|}{ Income } \\
\hline $2000-3000$ & $0.157^{\star}$ & $0.094^{*}$ & $0.097^{\star}$ \\
\hline$>3000$ & $0.205^{\star}$ & $0.108^{*}$ & $0.103^{*}$ \\
\hline Gender: female & 0.009 & -0.048 & -0.045 \\
\hline \multicolumn{4}{|l|}{ Education§ } \\
\hline Junior high school & $0.083^{*}$ & 0.037 & 0.034 \\
\hline Senior high school & 0.052 & 0.027 & 0.031 \\
\hline College and above & -0.012 & -0.028 & -0.031 \\
\hline Residence: urban & 0.061 & 0.021 & 0.002 \\
\hline $\begin{array}{l}\text { Living arrangement: empty } \\
\text { nester }\end{array}$ & $-0.086^{*}$ & -0.061 & -0.030 \\
\hline
\end{tabular}

Marital status

\begin{tabular}{|c|c|c|c|}
\hline Married & -0.079 & -0.064 & -0.057 \\
\hline Divorced/widowed & $-0.109^{*}$ & -0.066 & -0.054 \\
\hline Chronic disease: yes & & $-0.239^{*}$ & $-0.234^{*}$ \\
\hline \multicolumn{4}{|c|}{ Health service satisfaction†† } \\
\hline Dissatisfied & & 0.088 & 0.086 \\
\hline $\begin{array}{l}\text { Neither satisfied nor } \\
\text { dissatisfied }\end{array}$ & & $0.225^{\star}$ & $0.196^{*}$ \\
\hline Satisfied & & $0.524^{*}$ & $0.453^{*}$ \\
\hline Very satisfied & & $0.307^{\star}$ & $0.258^{*}$ \\
\hline Smoking: yes & & $-0.116^{\star}$ & $-0.110^{*}$ \\
\hline Physical activity: yes & & $0.078^{*}$ & $0.078^{*}$ \\
\hline \multicolumn{4}{|l|}{ Lonelinessł‡ } \\
\hline Moderate & & & $-0.190^{\star}$ \\
\hline Moderately high & & & $-0.260^{*}$ \\
\hline $\mathrm{F}$ & 6.007 & 12.874 & 15.246 \\
\hline $\mathrm{R}^{2}$ & 0.091 & 0.256 & 0.308 \\
\hline$\Delta \mathrm{R}^{2}$ & 0.091 & 0.165 & 0.052 \\
\hline
\end{tabular}

${ }^{*} \mathrm{p}<0.05$.

†Reference group $=60-69$.

$\ddagger$ Reference group $=<2000$.

$\S$ Reference group=primary school or below.

१Reference group=single.

†Reference group=very dissatisfied.

船eference group=low.

$\beta$, standardised regression coefficient; QOL, quality of life.

of our participants experienced a moderate or moderately high level of loneliness. The prevalence of loneliness found by us is comparable with that found for older people in other Chinese cities, such as Shanghai, ${ }^{930}$ but is considerably greater than that reported in the developed country Finland..$^{35}$ The substantial social and economic changes occurring in China along with the increase in the number of older people increases the likelihood that more older people will be lonely. ${ }^{18}{ }^{30}$ Therefore, there is an urgent need to perform interventions that will mitigate their loneliness. ${ }^{9}$

Consistent with other studies, ${ }^{12} 36$ suffering from a chronic disease(s) was an important risk factor for QOL among older people. Monitoring and ameliorating a chronic disease may reduce its negative impact on the QOL of the older population. ${ }^{2}$ However, representative data from six countries, including China, have indicated that effective healthcare coverage among older people with chronic diseases is between $20.7 \%$ and $48.2 \% .^{37}$ Given the continuous nature of chronic care, it should be more effective to provide the older people with healthcare services in community-based settings, for example, primary healthcare centres. ${ }^{2}$ The WHO study also urged that permanent mechanisms be established that would expand healthcare coverage for older people with chronic conditions. ${ }^{3738}$

We found that the QOL of our participants was positively correlated with their satisfaction with health services, which is consistent with the results of a related study. ${ }^{8}$ The older people who are more satisfied with their health services are more prone to regard the healthcare they receive as effective and hence adhere to the recommended treatment(s), which consequently may further improve their physical and mental health. ${ }^{8}$ This finding may explain why the older people who reported a greater degree of satisfaction with their healthcare also reported a better QOL. However, only half of our participants $(49.5 \%)$ were satisfied or very satisfied with their health services. Poor quality, high costs and poor accessibility to healthcare may be reasons for dissatisfaction with health services in China. ${ }^{9} 39$

Our study had several limitations. First, the study was conducted in only one Chinese city, which may partly limit representation of Chinese older people. Additional studies in other areas of China will help determine whether our findings can be generalised. Second, this study employed a cross-sectional design, which may limit the ability to confirm a causal relation between QOL and loneliness. A future study, applying a more effective design, such as a case-controlled design or cohort design, should be conducted to establish the casual relation between loneliness and QOL. Last, the unique use of a self-report response may cause bias, such as a consistency motif, acquiescence bias and social desirability. The two primary ways to control for these method biases through the design of the study's procedures and statistical controls should be undertaken in future research. ${ }^{40}$ In addition, our study identified multiple factors that may influence QOL in older people; these factors can be addressed separately and in depth in the future.

\section{CONCLUSIONS}

Our study provides information regarding the relation among QOL, loneliness and health-related characteristics of Chinese older people. Loneliness is a crucial problem 
facing the elderly. This study indicated that loneliness was negatively associated with QOL among older people. To address the findings of this study, it is suggested that we may need to be aware of the loneliness of older people and take action to minimise loneliness and improve QOL. Furthermore, managing chronic diseases and improving the health service may contribute to better QOL for older people.

Acknowledgements The authors wish to thank Xia Min for help with sample collection. We thank all of the participants in this study.

Contributors YZ wrote the manuscript. YZ and JL participated in the data collection and analysis. $Z Y$ collected the data. $Z Y$ and $B Q$ designed the study and revised the manuscript. $B Q$ obtained funding. All authors read and approved the final manuscript.

Funding This work was supported by the National Natural Science Foundation of China, grant number 71473269 and 71673301 .

Disclaimer The funders had no role in study design, data collection and analysis, decision to publish or preparation of the manuscript.

Competing interests None declared.

Patient consent Not required.

Ethics approval Ethical protocol was obtained from the Bioethics Advisory Commission of China Medical University. All participants provided written informed consent before participating in the study.

Provenance and peer review Not commissioned; externally peer reviewed.

Data sharing statement № additional unpublished data.

Open access This is an open access article distributed in accordance with the Creative Commons Attribution Non Commercial (CC BY-NC 4.0) license, which permits others to distribute, remix, adapt, build upon this work non-commercially, and license their derivative works on different terms, provided the original work is properly cited, appropriate credit is given, any changes made indicated, and the use is non-commercial. See: http://creativecommons.org/licenses/by-nc/4.0/.

\section{REFERENCES}

1. World Health Organization. WHO World report on health and ageing. 2015 http://101.96.8.164/apps. who.int/iris/bitstream/10665/186463/ 1/9789240694811_eng.pdf?ua=1 (Accessed 10 Oct 2017).

2. Shanghai Municipal Center for Disease Control \& Prevention (SCDC). Study on global AGEing and Adult Health Wave 1 China National Report. 2012 https://www.researchgate.net/publication/277305920 China_Study_on_global_AGEing_and_adult_health_SAGE_Wave_1_ National_Report (Accessed in 01 Oct 2017).

3. HelpAge International. HelpAge International Annual review 2015. 2015 http://www.helpage.org/who-we-are/annual-review-2015/\# looking-forward (Retrieved online 08 Oct 2017).

4. HelpAge International. Global age watch index. 2014 http://www. helpage.org/global-agewatch/about/about-global-agewatch/ (Retrieved online 14 Oct 2017).

5. Wang Z, Li X, Chen M. Catastrophic health expenditures and its inequality in elderly households with chronic disease patients in China. Int J Equity Health 2015;14:8.

6. World Health Organization. Active ageing: a policy framework. 2002 http://apps.who.int/iris/bitstream/10665/67215/1/WHO_NMH_NPH_ 02.8.pdf (Accessed in 01 Oct 2017).

7. Bowling A. The Psychometric Properties of the Older People's Quality of Life Questionnaire, Compared with the CASP-19 and the WHOQOL-OLD. Curr Gerontol Geriatr Res 2009;2009:1-12.

8. Zhang Y, Zhou Z, Gao J, et al. Health-related quality of life and its influencing factors for patients with hypertension: evidence from the urban and rural areas of Shaanxi Province, China. BMC Health Serv Res 2016;16:277.

9. Chen Y, Hicks A, While AE. Quality of life and related factors: a questionnaire survey of older people living alone in Mainland China. Qual Life Res 2014;23:1593-602.

10. Bélanger E, Ahmed T, Vafaei A, et al. Sources of social support associated with health and quality of life: a cross-sectional study among Canadian and Latin American older adults. BMJ Open 2016;6:e11503.
11. Cerin $\mathrm{E}$, Sit $\mathrm{CH}$, Zhang CJ, et al. Neighbourhood environment, physical activity, quality of life and depressive symptoms in Hong Kong older adults: a protocol for an observational study. BMJ Open 2016;6:e010384.

12. Cadore EL, Izquierdo M. Exercise interventions in polypathological aging patients that coexist with diabetes mellitus: improving functional status and quality of life. Age 2015;37:64.

13. Barile JP, Thompson WW, Zack MM, et al. Multiple chronic medical conditions and health-related quality of life in older adults, 20042006. Prev Chronic Dis 2013;10:E162.

14. Luanaigh CO, Lawlor BA. Loneliness and the health of older people. Int J Geriatr Psychiatry 2008;23:1213-21.

15. Liu LJ, Guo Q. Loneliness and health-related quality of life for the empty nest elderly in the rural area of a mountainous county in China. Qual Life Res 2007;16:1275-80.

16. Wilson RS, Krueger KR, Arnold SE, et al. Loneliness and risk of Alzheimer disease. Arch Gen Psychiatry 2007;64:234-40.

17. Cacioppo JT, Hawkley LC, Thisted RA. Perceived social isolation makes me sad: 5-year cross-lagged analyses of loneliness and depressive symptomatology in the Chicago Health, Aging, and Social Relations Study. Psychol Aging 2010;25:453-63.

18. Chen Y, Hicks A, While AE. Loneliness and social support of older people in China: a systematic literature review. Health Soc Care Community 2014;22:113-23.

19. Jakobsson U, Hallberg IR. Loneliness, fear, and quality of life among elderly in Sweden: a gender perspective. Aging Clin Exp Res 2005;17:494-501.

20. Verhagen I, Ros WJ, Steunenberg B, et al. Ethnicity does not account for differences in the health-related quality of life of Turkish, Moroccan, and Moluccan elderly in the Netherlands. Health Qual Life Outcomes 2014;12:138.

21. Luo Y, Waite LJ. Loneliness and mortality among older adults in China. J Gerontol B Psychol Sci Soc Sci 2014;69:633-45.

22. World Health Organization. Definition of an older or elderly person. 2010 http://www.who.int/healthinfo/survey/ageingdefnolder/en/ index.html (Accessed in 06 Jun 2018).

23. Cheng $\mathrm{P}$, Jin $\mathrm{Y}$, Sun $\mathrm{H}$, et al. Disparities in prevalence and risk indicators of loneliness between rural empty nest and nonempty nest older adults in Chizhou, China. Geriatr Gerontol Int 2015;15:356-64

24. Tan Z, Liang Y, Liu S, et al. Health-related quality of life as measured with EQ-5D among populations with and without specific chronic conditions: a population-based survey in Shaanxi Province, China. PLoS One 2013;8:e65958.

25. The WHOQOL Group. Development of the World Health Organization WHOQOL-BREF quality of life assessment. The WHOQOL Group. Psychol Med 1998;28:551-8.

26. Liu GG, Wu H, Li M, et al. Chinese time trade-off values for EQ-5D health states. Value Health 2014;17:597-604.

27. Sun S, Chen J, Johannesson M, et al. Regional differences in health status in China: population health-related quality of life results from the National Health Services Survey 2008. Health Place 2011;17:671-80.

28. Zhang T, Shi W, Huang Z, et al. Influence of culture, residential segregation and socioeconomic development on rural elderly healthrelated quality of life in Guangxi, China. Health Qual Life Outcomes 2016;14:98.

29. Deng $X$, Dong $P$, Zhang L, et al. Health-related quality of life in residents aged 18 years and older with and without disease: findings from the First Provincial Health Services Survey of Hunan, China. BMJ Open 2017;7:e015880.

30. Chen Y, Hicks A, While AE. Loneliness and social support of older people living alone in a county of Shanghai, China. Health Soc Care Community 2014;22:429-38.

31. Hoi leV, Chuc NT, Lindholm L. Health-related quality of life, and its determinants, among older people in rural Vietnam. BMC Public Health 2010;10:549.

32. Patel KV, Guralnik JM, Dansie EJ, et al. Prevalence and impact of pain among older adults in the United States: findings from the 2011 National Health and Aging Trends Study. Pain 2013;154:2649-57.

33. Bhattarai P, Phillips JL. The role of digital health technologies in management of pain in older people: An integrative review. Arch Gerontol Geriatr 2017;68:14-24.

34. Hawkley LC, Thisted RA, Cacioppo JT. Loneliness predicts reduced physical activity: cross-sectional \& longitudinal analyses. Health Psychol 2009;28:354-63.

35. Routasalo PE, Savikko N, Tilvis RS, et al. Social contacts and their relationship to loneliness among aged people - a population-based study. Gerontology 2006;52:181-7. 
36. Venturelli $\mathrm{M}$, Cè E, Limonta $\mathrm{E}$, et al. Effects of endurance, circuit, and relaxing training on cardiovascular risk factors in hypertensive elderly patients. Age 2015;37:101.

37. Goeppel C, Frenz P, Tinnemann P, et al. Universal health coverage for elderly people with non-communicable diseases in low-income and middle-income countries: a cross-sectional analysis. Lancet 2014;384:S6
38. Trivedi AN, Moloo H, Mor V. Increased ambulatory care copayments and hospitalizations among the elderly. N Engl J Med 2010;362:320-8.

39. Eggleston $\mathrm{K}$, Ling $\mathrm{L}$, Qingyue $\mathrm{M}$, et al. Health service delivery in China: a literature review. Health Econ 2008;17:149-65.

40. Podsakoff PM, MacKenzie SB, Lee JY, et al. Common method biases in behavioral research: a critical review of the literature and recommended remedies. J Appl Psychol 2003;88:879-903. 